\title{
ANÁLISE DE FREQUÊNCIA DE PRECIPITAÇÃO E CARACTERIZAÇÃO DE ANOS SECOS E CHUVOSOS PARA A BACIA DO RIO ITAJAÍ
}

\author{
GOUVEA, Regina Luiza- gouveareginaluiza@gmail.com \\ Centro Educacional Leonardo da Vinci / UNIASSELVI
}

CAMPOS, Cláudia Camargo - claudia.campos@udesc.br

Universidade do Estado de Santa Catarina / UDESC

MENEZES, João Thadeu - thadeu@univali.br

Universidade do Vale do Itajaí / UNIVALI

MOREIRA, Genésio Freitas - genesiomoreira@gmail.com
Universidade Federal de Lavras / UFLA

\begin{abstract}
RESUMO: Eventos extremos de precipitação influenciam a sociedade de forma direta e indireta. Dependendo de sua magnitude e extensão, os danos gerados por estes eventos podem, na maioria das vezes, ser enquadrados na categoria de desastres naturais. 0 objetivo deste trabalho é estabelecer e discutir valores normais e extremos para a chuva na bacia do rio Itajaí, através da utilização da técnica estatística dos quantis, utilizando dados de precipitação das estações convencionais da Agência Nacional de Águas (ANA), no período de 1930-2013. O cálculo de frequência de classes de chuva foi realizado empregando-se as classes recomendadas pelo Instituto Nacional de Meteorologia (INMET). A análise de frequência mostrou que a classe de precipitação $0,1-2,5 \mathrm{~mm}$ é a mais comum, com ocorrência superior a $14,5 \%$. As classes entre $50-100 \mathrm{~mm}$ e $>100 \mathrm{~mm}$ não chegam a ultrapassar $5 \%$ de frequência. Quanto aos anos com os maiores acumulados anuais, destacam-se os anos de 1983 e 1998 e 2008.
\end{abstract}

PALAVRAS-CHAVES: Extremos de precipitação; análise de frequência; quantil.

ANALYSIS OF PRECIPITATION FREQUENCY AND CHARACTERIZATION OF DRY AND RAINY YEARS AT THE ITAJAÍ RIVER BASIN

\begin{abstract}
Extreme precipitation events influence society directly and indirectly. Depending on their magnitude and extent, the impacts generated by events can, for the most part, fall into the category of natural disasters. The aim of this work is to establish and discuss normal and extreme values for rainfall in the Itajaí river basin, using the statistical technique of quantiles and rainfall data from the National Meteorological Agency (ANA) in the period of 1930-2013. The calculation of frequency of rainfall classes was performed using the classes recommended by the National Institute of Meteorology (INMET). Frequency analysis showed that the class of precipitation $0.1-2.5 \mathrm{~mm}$ is the most common, with an occurrence higher than $14.5 \%$. Classes between $50-100 \mathrm{~mm}$ and> $100 \mathrm{~mm}$ do not exceed $5 \%$ of frequency. As for the years with the highest annual accumulations, the years of 1983, 1998 and 2008 stand out.
\end{abstract}

KEYWORDS: Extreme Precipitation; frequency analysis, quantile.

\section{INTRODUÇÃO}

A ocorrência da precipitação é determinada pela gênese e pelo desenvolvimento de fenômenos atmosféricos em diferentes escalas espaciais e temporais e de suas interações com a superfície terrestre por meio de trocas de fluxos de calor e umidade (CALVETTI et al. 2006). E um dos elementos meteorológicos que exerce maior influência sobre as condições ambientais, com efeitos diretos sobre o balanço hídrico (SORIANO e GALDINO, 2002). No caso da 
região Sudeste do Brasil, a mais populosa e economicamente mais desenvolvida, períodos longos de seca podem afetar dramaticamente algumas culturas na região, causando prejuízos econômicos, assim como precipitações intensas e persistentes por muitos dias podem gerar danos econômicos e perdas de vidas, especialmente nos locais de maior vulnerabilidade (CARVALHO e JONES, 2009).

Não se deve atribuir a responsabilidade pelos prejuízos socioeconômicos aos elementos climáticos, mesmo porque, como afirma Monteiro et al. (2012), a opção por uma ocupação do solo, muitas vezes, desconsidera as vulnerabilidades do local escolhido para se estabelecer, portanto a compreensão destes elementos é fundamental no planejamento urbano e agrícola.

Segundo Burges et al. (2015), na Jamaica, a análise da precipitação extrema é importante para projetos de infraestrutura de controle de inundações, especialmente com a possibilidade de aumento das intensidades sob mudanças climáticas. De acordo com os autores, registros de extremos de precipitação no país incluem eventos que estão entre as maiores medições conhecidas de precipitação em nível global. Segundo Noriah e Rakhecha (2001), em Selangor, Malásia, as precipitações são influenciadas pelas monções de sudoeste e nordeste, com precipitações máximas ocorrendo durante os meses entre as monções, devido às tempestades severas frequentes. Os maiores valores diários de precipitação para o sudeste da Malásia variam de mais que $250 \mathrm{~mm}$ ao longo da costa a $175 \mathrm{~mm}$ no interior.

Em áreas urbanas, o estudo de chuvas intensas e/ou prolongadas tornase vital, devido aos incidentes de variadas proporções que podem ocasionar, como as inundações e escorregamentos, alagamentos temporários, colapso de serviços de infraestrutura, perdas de vidas humanas, além dos danos à saúde psicológica, devido ao stress causado pelas situações enfrentadas em momentos de inundações (SOUZA et al. 2012). Episódios de chuvas com intensidades superiores a $60 \mathrm{~mm}$ ocorridos em 24 horas foram avaliados por Zanella (2007), devido aos danos que causam em áreas urbanas. Estudos semelhantes foram realizados por Gonçalves (1992) para a cidade de Salvador. Souza et al. (2012) avaliaram dados de precipitação diária para determinar e classificar limiares da precipitação pluviométrica, na cidade do Recife. Os resultados mostraram que quando há registros de precipitação dentro das classes de chuva muito forte $(18,6-55,3 \mathrm{~mm})$ e forte $(\geq 55,3 \mathrm{~mm})$, há escorregamentos e muitos pontos de alagamentos.

Neste contexto, o emprego de metodologias que permitam compreender o comportamento da precipitação e sua oscilação anual, torna-se indispensável para o planejamento urbano e agrícola da região. Assim, esta pesquisa tem com objetivo estabelecer e discutir valores normais e extremos para a chuva na bacia do rio Itajaí, através da utilização da técnica estatística dos quantis. Por se tratar de uma região onde a forma da bacia e a declividade dos cursos d'água favorecem a ocorrência de inundações é que se propõe conhecer a concentração da precipitação em diferentes intervalos de classes de intensidade e a frequência com que ocorrem na bacia do rio Itajaí.

\section{METODOLOGIA}

Foram selecionadas dezenove séries pluviométricas, das quarenta e oito disponibilizadas no sistema de informações hidrológicas Hidroweb para a 
classificação da intensidade de chuva em intervalos de classes. Os critérios de seleção adotados foram séries históricas com períodos de dados superiores a 30 anos, conforme padronização da Organização Meteorológica Mundial (OMM). Para a análise de frequência, foram utilizados dados de 1930 e 2013 a fim de compará-los aos anos chuvosos.

Foram empregados dados de chuva consistidos disponibilizados até o ano de 2006. No período que compreende os anos de 2007 e 2013, a análise de consistência foi realizada utilizando o programa HIDRO-PLU, da Agência Nacional de Energia Elétrica (ANEEL). O modelo admite a utilização de um número máximo de nove estações de apoio, estabelecendo intervalos de aceitação. Se o total mensal ou anual da estação em análise estiver fora do intervalo de aceitação de todas as estações de apoio selecionadas para os testes, o programa recusa este valor e sugere outro com maior possibilidade de ocorrência. Se o total mensal da estação em análise ficar dentro do intervalo de aceitação de uma das estações de apoio selecionadas para os testes, o valor é aceito e não é sugerido outro valor (ANELL, 2017).

A localização das estações pluviométricas na bacia do rio Itajaí encontrase na Figura 1. Os códigos, municípios, coordenadas geográficas, altitude e período das séries selecionadas são apresentados na Tabela 1.

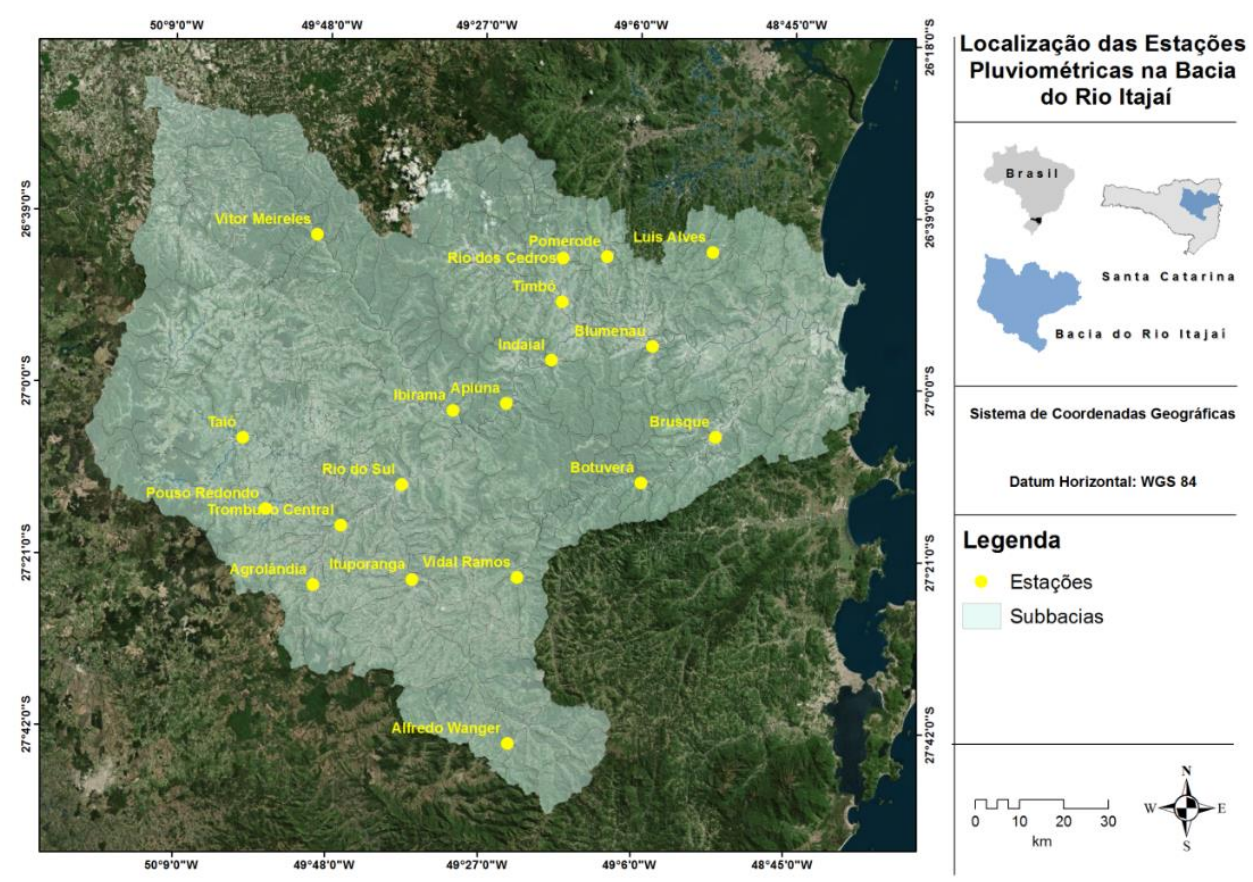

Figura 1- Distribuição espacial das estações pluviométricas da bacia do Rio Itajaí

Tabela 1- Localização das estações pluviométricas da bacia do rio Itajaí.

\begin{tabular}{cccc}
\hline Estação Código Latitude Longitude $\begin{array}{c}\text { Altitude } \\
(\mathbf{m})\end{array}$ \\
\hline
\end{tabular}




\begin{tabular}{lcccc} 
Agrolândia & 2749041 & $-27,4114$ & $-49,8314$ & 850 \\
Alfredo Wagner & 2749007 & $-27,7306$ & $-49,3828$ & 550 \\
Apiúna & 2749000 & $-27,0381$ & $-49,395$ & 93 \\
Blumenau & 2649007 & $-26,9181$ & $-49,0653$ & 12 \\
Botuverá & 2749045 & $-27,1967$ & $-49,0872$ & - \\
Brusque & 2748000 & $-27,1008$ & $-48,9181$ & 27 \\
Ibirama & 2749001 & $-27,0539$ & $-49,5167$ & 151 \\
Indaial & 2649001 & $-26,9492$ & $-49,2939$ & 76 \\
Ituporanga & 2749002 & $-27,3986$ & $-49,6058$ & 370 \\
Luiz Alves & 2648002 & $-26,7242$ & $-48,9317$ & 90 \\
Pomerode & 2649002 & $-26,7367$ & $-49,1703$ & 63 \\
Pouso Redondo & 2749006 & $-27,2572$ & $-49,9408$ & 353 \\
Rio do Sul & 2749039 & $-27,2056$ & $-49,6317$ & 350 \\
Rio dos Cedros & 2649008 & $-26,7408$ & $-49,2706$ & 80 \\
Taió & 2749003 & $-27,1131$ & $-49,9944$ & 360 \\
Timbó & 2749004 & $-26,8297$ & $-49,2719$ & 400 \\
Trombudo & 2749013 & $-27,2903$ & $-49,7689$ & 350 \\
Central & 2749033 & $-27,3925$ & $-49,3656$ & 550 \\
Vidal Ramos & & & & \\
\hline & & & \\
\hline
\end{tabular}

A análise de frequência de dias com chuva foi realizada na planilha eletrônica do programa Microsoft Office Excel $($. O cálculo foi feito empregandose as classes recomendadas pelo INMET: 0,1-2,5 mm, 2,5-5 mm, 5-10 mm, 10$15 \mathrm{~mm}, 15-25 \mathrm{~mm}, 25-50 \mathrm{~mm}, 50-100 \mathrm{~mm} \mathrm{e}>100 \mathrm{~mm}$. As classes de frequência a partir de $100 \mathrm{~mm}$ foram subdivididas em intervalos de 50, constituindo-se as seguintes classes: $100-150$ mm, 150-200 mm, 200-250 mm, 250-300 mm, 300-350 mm e $>350 \mathrm{~mm}$. A precipitação diária foi dividida nas seguintes classes: chuvisco (0,1 a 2,5 mm), chuva fraca $(2,5$ a 10,0 mm), chuva moderada $(10,0$ a $15,0 \mathrm{~mm})$, chuva relativamente forte (15 a $25 \mathrm{~mm}$ ) chuva forte $(25,0$ a $50,0 \mathrm{~mm}$ ) e chuva extrema (acima de $50 \mathrm{~mm}$ ).

Para a caracterização dos anos secos e chuvosos, foi utilizada a técnica dos quantis, empregada para separar as distribuições da amostra. Um quantil de ordem $p$ é um valor numérico que divide a distribuição em duas partes separando uma amostra em duas massas de observações numéricas, com $100 \times$ p dos elementos localizados à esquerda do quantil "amostral" e os demais $100 \times(1-p) \%$, à direita. Para o Nordeste brasileiro uma série de trabalhos foi realizada por Xavier e Xavier (1984); Xavier e Xavier (1987), Xavier e Xavier (1990); Xavier et al.(2002) com o objetivo de classificar e monitorar anos secos e chuvosos.

As séries históricas de precipitação foram organizadas na planilha eletrônica do programa Microsoft Exce ${ }^{\circledR}$, agrupando em uma única tabela os totais de chuva acumulada em cada mês de cada ano da série histórica. Em seguida, os valores foram somados para obtenção do total pluviométrico acumulado durante cada ano. Posteriormente, os valores anuais foram ordenados, em ordem crescente, para aplicação da técnica estatística. Com os valores ordenados em uma única tabela foram determinados para os respectivos 
quantis. A escolha dos quantis pode variar de acordo com o objetivo da pesquisa. Neste estudo, foram utilizados o quantil de ordem $5 \%$ (valores extremos inferiores para chuva acumulada em determinado ano) e o quantil de ordem $95 \%$ (valores extremos superiores para a chuva acumulada em determinado ano). Isso representa que dos totais acumulados em cada ano da série histórica, ordenados do menor valor para o maior valor, $5 \%$ destes valores estarão abaixo do $\mathrm{Q}_{(0,05)}$, ou seja, representarão valores extremos de chuva (baixa pluviosidade) no outro extremo da tabela, os valores acima do $\mathrm{Q}_{(0,95)}$ representam os valores extremos superiores de chuva (elevada pluviosidade).

Mediante os quantis, foram definidos os intervalos correspondentes aos eventos Extremamente Seco (ES), Muito Seco (MS), Seco (S), Normal (N), Chuvoso (C), Muito Chuvoso (MC) e Extremamente Chuvoso (EC), representadas pelos seus respectivos quantis $\mathrm{Q}_{(0,5)}, \mathrm{Q}_{(0,15),} \mathrm{Q}_{(0,35)}, \mathrm{Q}_{(0,50)}, \mathrm{Q}_{(0,65)} \mathrm{e}$ $\mathrm{Q}_{(0,85),} \mathrm{Q}_{(95)}$ para cada uma das séries históricas, onde, $\mathrm{Q}$ significa o limite do quantil adotado para a realização dos cálculos deste trabalho. A descrição segue o método aplicado por Xavier e Xavier (1999).

Define-se o quantil Q, para cada número real p entre 0 (zero) e 1 (um), como valor em milímetros de chuva que satisfaça a condição:

$\operatorname{Prob}\left(X \leq Q_{p}\right)=p$

Equação (1)

Consequentemente, se $F=F_{x}$, for a função de distribuição, ou função de repartição, de probabilidades associada à variável aleatória contínua $X$, admitindo uma "inversa" $F^{-1}$, num intervalo $(a, b),-\infty \leq a<b \leq+\infty$, tal que $F(a)=0$ e $F(b)=1$, então:

$F\left(Q_{p}\right)=p$,

Equação (21)

ou equivalente,

$\mathrm{F}^{-1}(\mathrm{p})=\mathrm{Q}_{\mathrm{p}}$

Equação (3)

Ademais, para qualquer valor observado $\mathrm{X}_{0}$ da precipitação, o número $\mathrm{p}_{0}$ $\left(0<\right.$ po $\left._{0}<1\right)$, tal que:

$F\left(X_{0}\right)=p_{0}$,

Equação (4)

Diz-se ordem quantília po associada a $\mathrm{X}_{0}$.

De acordo com Xavier e Xavier (1999), uma interpretação simples para o quantil Qp é a seguinte: supondo a "probabilidade" p expressada em termos porcentuais, espera-se que em $\mathrm{p}(\%)$ dos anos a medida da chuva $X$ não deve ultrapassar o valor desse quantil Qp, em milímetros, enquanto para (100 - p) \% dos anos tal valor será excedido.

Os totais de chuva acumulada em cada mês de cada ano da série histórica foram agrupados em uma única tabela e somados para aquisição do total pluviométrico acumulado durante cada ano. Em seguida, os valores anuais foram ordenados, do menor para o maior, para a aplicação da técnica estatística e estabelecimento dos valores dos quantis

\section{RESULTADOS E DISCUSSÃO}

A análise de frequência de classes de precipitação diária realizada para as séries pluviométricas da bacia do rio Itajaí (Figura 2) mostra que a classe 0,1$2,5 \mathrm{~mm}$ é a mais comum, com ocorrência superior a 14,5\%. Neste intervalo de 
classe, a estação pluviométrica de Trombudo Central foi a que apresentou maior ocorrência, com $36 \%$. As classes com valores de precipitação entre 2,5 mm e 5 $\mathrm{mm}$ possuem frequência em torno de $14,0 \%$, destacando-se a classe entre 5$10 \mathrm{~mm}$ de chuva diária, com valores que chegam a 22,0 \%. Estes resultados convergem com os encontrados por Salgado et al. (2007), para o município de Angra dos Reis, RJ, onde os valores de chuva entre 5-10 mm são mais expressivos que a classe entre 2,5-5 mm e maior ocorrência das classes com valores entre $0,1-2,5 \mathrm{~mm}$.

As classes de precipitação diária entre a 25-50 mm (chuva forte), apresentam frequências superiores às anteriores, com exceção da série pluviométrica de Vitor Meireles. As classes de 50-100 mm e >100 mm não chegam a ultrapassar $5 \%$ de frequência. Os resultados estão de acordo com o esperado, sendo os maiores volumes concentrados abaixo de $50 \mathrm{~mm}$, no entanto o que se destaca é existência dos valores diários extremos de chuva. Neste caso, mesmo que sejam esporádicos, podem apresentar efeitos negativos consideráveis, principalmente se forem posteriores a dias consecutivos de chuva.

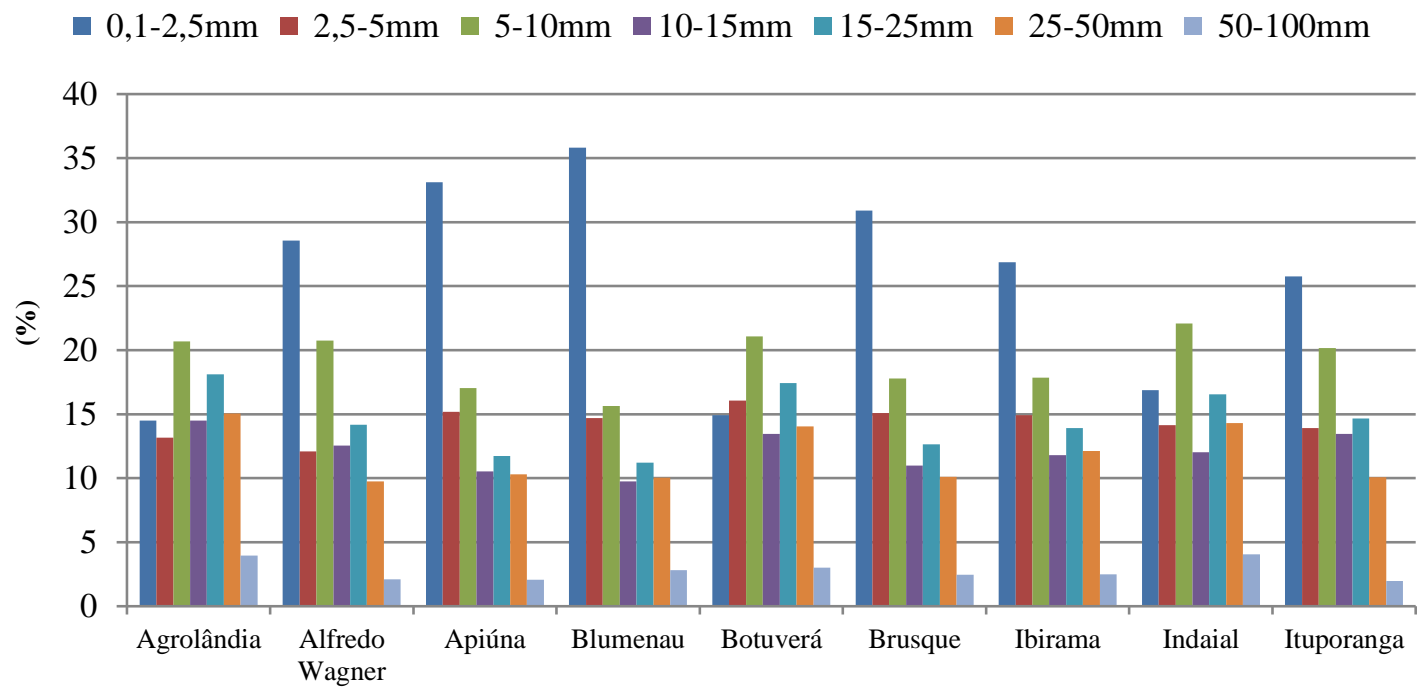




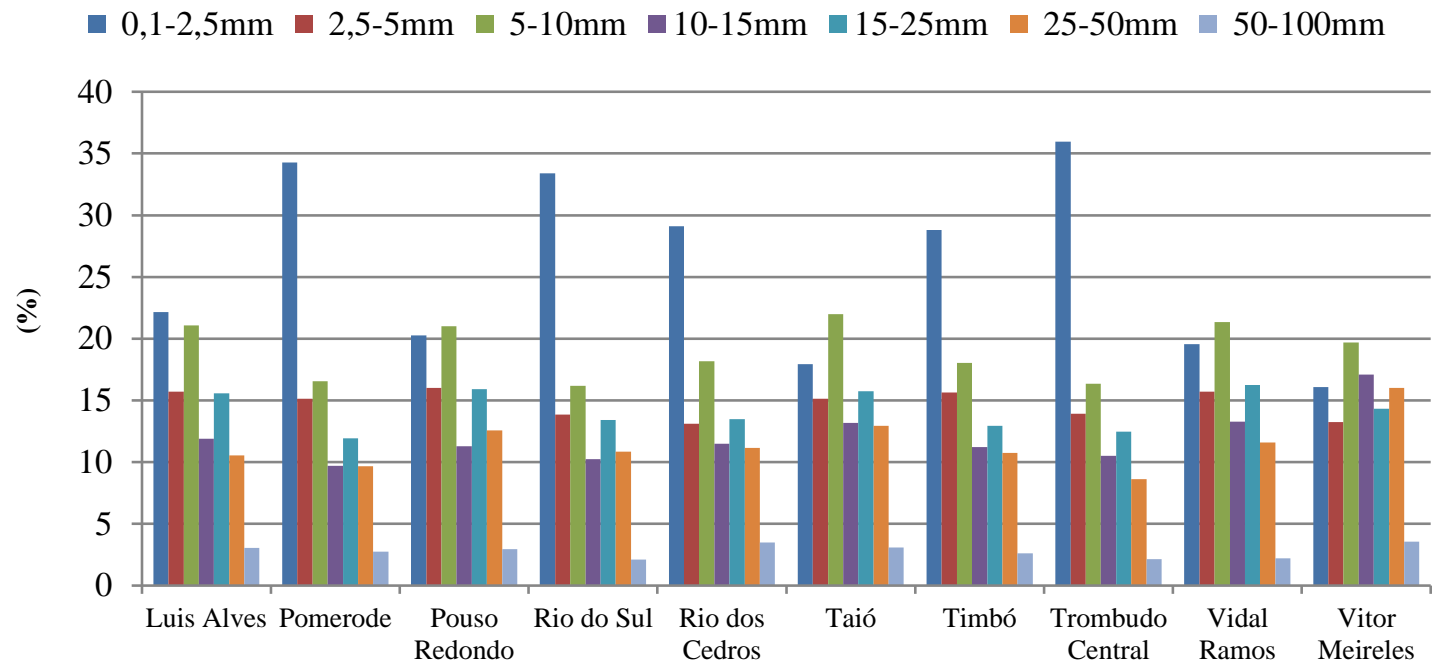

Figura 2- Frequência relativa (\%) de classes de precipitação para as séries históricas das estações meteorológicas situadas na bacia do rio Itajaí.

A Tabela 3 apresenta a subdivisão da classe de precipitação $>100 \mathrm{~mm}$ em números absolutos. A análise desses dados evidencia que as maiores frequências de precipitação entre 100 e 150 mm encontram-se nas estações de Luiz Alves, rio dos Cedros e Pomerode, contrastando com as frequências apresentadas nas estações de rio do Sul, Trombudo Central e Botuverá. As maiores frequências de extremos de chuva das classes entre 150-200 mm foram encontradas nas estações pluviométricas de Pomerode e Luiz Alves, 4 e 3, respectivamente. Para as classes de frequência entre 200-250 mm foram encontrados extremos nas estações de Blumenau, Luiz Alves e Pomerode. 0 maior total pluviométrico diário encontrado foi da estação pluviométrica de Blumenau com 250,9 mm no dia 24 de novembro de 2008, sengundo registros da ANA.

Tabela 3- Frequência absoluta de classes de precipitação para séries históricas das estações situadas na bacia do rio Itajaí.

\begin{tabular}{lcccc}
\hline \multirow{2}{*}{$\begin{array}{c}\text { Estações } \\
\text { pluviométricas }\end{array}$} & \multicolumn{2}{c}{ Classes de precipitação ( $\mathbf{m m})$} \\
\cline { 2 - 5 } & $\mathbf{1 0 0 - 1 5 0}$ 150-200 200-250 250-300 \\
\hline Agrolândia & 6 & & & \\
Alfredo Wagner & 11 & & 1 & 1 \\
Apiúna & 14 & & \\
Blumenau & 20 & 1 & \\
Botuverá & 4 & 1 & \\
Brusque & 13 & 2 & \\
Ibirama & 12 & & \\
Indaial & 11 & 1 & \\
Ituporanga & 13 & &
\end{tabular}




\begin{tabular}{lccc} 
Luiz Alves & 35 & 3 & 1 \\
Pomerode & 27 & 4 & 1 \\
Pouso Redondo & 11 & & \\
Rio do Sul & 3 & & \\
Rio dos Cedros & 28 & & \\
Taió & 14 & 1 & \\
Timbó & 18 & 2 & \\
Trombudo Central & 4 & 2 & \\
Vidal Ramos & 11 & & \\
Vitor Meireles & 5 & & \\
\hline
\end{tabular}

Eli et al. (2012) utilizaram dados do período de 1987 a 2008, da estação de Ituporanga, para a análise de frequência da precipitação. A média da precipitação anual foi de $1.606,3 \mathrm{~mm}$ e média mensal de $133,9 \mathrm{~mm}$. As estações do ano mais chuvosas foram o verão e a primavera, correspondendo a $59,6 \%$ da precipitação anual. A maior frequência de precipitação observada ficou dentro do intervalo de $1.719,8$ a $1.875,8 \mathrm{~mm}$. Para a estação de rio do Sul, Eli et al. (2013) encontraram média anual de $1.458,7 \mathrm{~mm}$ e média mensal de $1.21,56 \mathrm{~mm}$. As estações do ano mais chuvosas foram o verão e a primavera, correspondendo a 57,2 \% da precipitação anual.

Neste estudo, os valores referentes às altas e baixas pluviosidades foram obtidos através da técnica dos quantis apresentados na Tabela 4. De acordo com os totais acumulados em cada ano da série histórica, $5 \%$ destes valores estão abaixo do $\mathrm{Q}(0,05)$, ou seja, representam valores extremos de chuva (baixa pluviosidade). No outro extremo, os valores acima do $Q(0,95)$ representam os extremos superiores de chuva (elevada pluviosidade). As classes extremas (Extremamente Seco e Extremamente Chuvoso) apresentam, dessa forma, um intervalo relativamente menor (5\%), enquanto que a classe Normal apresenta o maior intervalo (30\%), compreendendo os valores entre 35 $\%$ e $65 \%$, conforme esperado. Os intervalos percentuais de cada quantil representam as probabilidades esperadas para cada um dos eventos que podem vir a ocorrer na série temporal, supondo que as características da precipitação sejam mantidas. Neste caso, nas séries mais longas obteve-se cinco anos de valores extremos de chuva acumulada no ano, enquanto nas séries mais curtas obteve-se apenas um da série estudada, tanto para baixa pluviosidade (anos extremamente secos), como para elevada pluviosidade (anos extremamente chuvosos).

Tabela 4- Classificação dos valores de chuva acumulada das séries históricas, na bacia, e valores estabelecidos para os quantis na bacia do rio Itajaí.

\begin{tabular}{ccccc}
\hline & $\leftarrow$ & & $\leftrightarrow$ & $\leftrightarrow$ \\
\multirow{2}{*}{ Classes } & $\mathbf{5 \%}$ & & $\mathbf{1 5 \%}$ & $\mathbf{3 5 \%}$ \\
& Ext. & & Muito & Seco \\
\hline \multirow{2}{*}{ Estação } & Seco & & Seco & \\
\hline
\end{tabular}




\begin{tabular}{lll}
\hline Agrolândia & 1110,9 & 1293,1 \\
Alfredo & 1101,7 & 1278,7 \\
Wagner & 936,1 & 1219,6 \\
Bluména & 1114,6 & 1289,6 \\
Botuverá & 1000,3 & 1299 \\
Brusque & 1131,1 & 1446,4 \\
Ibirama & 991,6 & 1191,9 \\
Indaial & 939,2 & 1254,7 \\
Ituporanga & 990,7 & 1179,4 \\
Luiz Alves & 1245,7 & 1455,9 \\
Pomerode & 1264,9 & 1458 \\
Pouso & 954,5 & 1122,1 \\
Redondo & 1076,6 & 1251,6 \\
Rio do Sul & 1146,5 & 1304,4 \\
Rio dos & 1045,1 & 1216,5 \\
Cedros & 1221,7 & 1293,4 \\
Taió & 999,6 & 1182,3 \\
Timbó & 1045,5 & 1114,1 \\
Trombudo & 962,3 & 1135,1 \\
Central & Vidal Ramos &
\end{tabular}

Tabela 5- Classificação dos valores de chuva acumulada das séries históricas, na bacia, e valores estabelecidos para os quantis na bacia do rio Itajaí.

\begin{tabular}{|c|c|c|c|c|c|}
\hline Classes & $\begin{array}{c}\leftrightarrow \\
50 \% \\
\text { Normal }\end{array}$ & & $\begin{array}{c}\leftrightarrow \leftrightarrow \\
\text { 65\% } \\
\text { Chuvoso }\end{array}$ & $\begin{array}{c}\leftrightarrow \\
85 \% \\
\text { Muito } \\
\text { Chuvoso }\end{array}$ & $\begin{array}{c}\overrightarrow{ } \\
95 \% \\
\text { Ext. } \\
\text { Chuvoso }\end{array}$ \\
\hline Estação & & $\begin{array}{l}\mathrm{Q}_{(0,65)} \\
(\mathrm{mm}) \\
\end{array}$ & $\begin{array}{l}Q_{(0,85)} \\
(\mathrm{mm})\end{array}$ & & $\begin{array}{l}\mathrm{Q}_{(0,95)} \\
(\mathrm{mm})\end{array}$ \\
\hline Agrolândia & 1653,2 & 1772,3 & 1955 & & 2304 \\
\hline $\begin{array}{l}\text { Alfredo } \\
\text { Wagner }\end{array}$ & 1560,4 & 1647,5 & 1849,1 & & 2065 \\
\hline Apiúna & 1472,7 & 1655,3 & 1854,4 & & 2038,8 \\
\hline Blumenau & 1630 & 1722,7 & 1928,6 & & 2405,7 \\
\hline Botuverá & 1546,7 & 1706,3 & 2004,7 & & 2296,6 \\
\hline
\end{tabular}




$\begin{array}{lrrll}\text { Brusque } & 1618,6 & 1717,3 & 1992,4 & 2274,8 \\ \text { Ibirama } & 1464,8 & 1516,9 & 1803,6 & 2035 \\ \text { Indaial } & 1637,2 & 1673,3 & 2005,7 & 2609,9 \\ \text { Ituporanga } & 1504,6 & 1596 & 1793 & 2112 \\ \text { Luiz Alves } & 1678,2 & 1882,4 & 2249,9 & 2635,4 \\ \text { Pomerode } & 1811,7 & 1919,8 & 2150,7 & 2471,9 \\ \text { Pouso } & 1476,7 & 1598,5 & 1732,9 & 2064,5 \\ \text { Redondo } & 1528,8 & 1666 & 1888,2 & 2305,8 \\ \text { Rio do Sul } & 150,9 & 2504,4 \\ \begin{array}{l}\text { Rio dos } \\ \text { Cedros }\end{array} & 1646,9 & 1774 & 2031,1 & 2051,5 \\ \text { Taió } & 1522,9 & 1645,8 & 1856,5 & 2064,1 \\ \text { Timbó } & 1618,1 & 1709,8 & 1898,3 & 2028,2 \\ \begin{array}{l}\text { Trombudo } \\ \text { Central }\end{array} & 1452,3 & 1554,2 & 1757 & 1874 \\ \text { Vidal Ramos } & 1366,9 & 1562,4 & 1665,4 & 2208 \\ \text { Vitor } & 1526,7 & 1608 & 1823 & \\ \text { Meireles } & & & \end{array}$

Acumulado de chuva de $1.811,7 \mathrm{~mm}$ representa pluviosidade normal para a localidade de Pomerode, enquanto para a estação pluviométrica de Vidal Ramos um acumulado anual de $1.874 \mathrm{~mm}$ representa um valor de alta pluviosidade. Os maiores valores do $\mathrm{Q}(0,95)$ foram observados nas estações pluviométricas de Luiz Alves e Indaial (2.635,4 e 2.609,9 mm).

Dentre os anos com os maiores valores pluviométricos (1983 e 1998) correspondem a anos de El Niño muito forte (1982-83 e 1997-98), contudo os anos de 1983 e 2008 foram os anos com os maiores volumes de chuva, sendo 2008 uma exceção à regra por se tratar de um ano de La Niña.

No sul do Brasil, extremos de precipitação podem ser atribuídos à influência da Oscilação Decadal do Pacífico. Estes eventos persistem por 20 a 30 anos e se assemelham aos eventos El Niño/La Niña, apresentando uma fase quente e uma fase fria. A fase fria caracteriza-se por anomalias negativas de Temperatura da Superfície do Mar (TSM) no Pacífico Tropical e, concomitantemente, anomalias de TSM positivas no Pacífico Extratropical no Hemisfério Norte e no Hemisfério Sul (STRECK, 2009). Durante o episódio ENOS 1982-83, a região Sul foi afetada com precipitação pluvial acima da normal durante o mês de julho (NERY, 2005). Em 1998, caracterizado como um ano de El Niño muito intenso, a região Sul recebeu excesso de precipitação durante a primavera e verão. Segundo Oliveira e Satyamurty (1998), o Sul do país recebeu, em média, $50 \mathrm{~mm} / \mathrm{mês}$ de chuva a mais que o normal durante outubro/97 - maio/98.

Segundo Dias et al. (2009), do ponto de vista meteorológico, a tragédia em Santa Catarina em 2008 foi o resultado de uma combinação de sistemas de diferentes escalas espaciais e temporais. Os autores ressaltam que, em escala global, os meses de setembro, outubro e novembro, assim como as primeiras semanas de dezembro, foram caracterizados pela presença de fortes anomalias 
de pressão atmosférica, de sinal alternado, em praticamente todo o Hemisfério Sul. Um anticiclone de bloqueio no oceano Atlântico determinou a ocorrência de ventos de leste sobre boa parte da costa da região Sul, transportando umidade do oceano para o continente, incidindo mais diretamente sobre o litoral de Santa Catarina. De acordo com os referidos autores, os maiores volumes de chuva do país ocorreram no estado de Santa Catarina, principalmente, na região litorânea e no Vale do Itajaí, com as precipitações fracas, porém persistentes, durante três meses. Há que se destacar, de acordo com os autores, que quando um sistema de bloqueio atmosférico se estabelece, as condições do tempo persistem quase sem variações até que o sistema se desloque ou dissipe. Em Blumenau, no mês de novembro de 2008, foram registrados $1.002 \mathrm{~mm}$ de chuva, superando o recorde anterior de $578,8 \mathrm{~mm}$ (jan 1989) e os $542,2 \mathrm{~mm}$ precipitados no mês de julho de 1983.

O ano de 1983 foi classificado com Extremamente Chuvoso em todas as estações pluviométricas analisadas e também o ano com maior total anual, exceto para as estações de Apiúna e Luiz Alves, que apresentaram um maior total anual em 2008 (2.870,2 e 2.905,7 mm, respectivamente) e Ibirama, com acumulado anual de 2734,5 em 1990. Para estas estações, o segundo maior total anual foi o ano 1983, com 2.159,5 mm em Apiúna, 2.896,2 mm em Luiz Alves e $2.508,4 \mathrm{~mm}$ em Ibirama. Nas demais localidades da bacia, os totais acumulados ultrapassaram $2.000 \mathrm{~mm}$ de precipitação, superando os $3.000 \mathrm{~mm}$ em Brusque e Pomerode, com 3.003,8 mm e 3.010,8 mm, respectivamente. Este ano totalizou 196 extremos de chuva com valores diários superiores a 50 $\mathrm{mm}$. Os maiores valores foram observados em Rio dos Cedros, com 17 extremos e Luiz Alves e Vitor Meireles apresentaram 16 e 15 extremos diários de precipitação, respectivamente. Os menores valores de extremos para este ano foram observados em Ibirama e Vidal Ramos, com 5 extremos.

Os anos de 1990 e 1998 foram classificados como ano Muito Chuvoso ou Extremamente Chuvoso para as estações pluviométricas de Timbó, Pomerode, Pouso Redondo, Trombudo Central, Vitor Meireles, Rio do Sul e Indaial. Nas demais estações pluviométricas, o ano de 1990 ou 1998 aparece como ano Muito Chuvoso ou Extremamente Chuvoso, com exceção de Alfredo Wagner, onde 1990 e 1998 não figuram entre os mais chuvosos. Em 1990 não há registro de extremos em Alfredo Wagner por predominar precipitações inferiores a $50 \mathrm{~mm}$. Em 1990, os maiores valores de extremos foram observados em Ituporanga (10). Em 1998, o excesso de chuvas, decorrente do fenômeno El Niño, afetou a agricultura catarinense, provocando queda no rendimento físico e perdas de qualidade na produção agrícola (TERACINES, 2000).

Apesar do evento de 2008, em Santa Catarina, ter proporcionado uma elevada quantidade de chuva, que devido à sua continuidade ocasionou inundações e movimentos de massa, este não foi caracterizado como um ano Muito Chuvoso ou Extremamente Chuvoso na maior parte das séries pluviométricas analisadas. Este ano classifica-se como Extremamente Chuvoso somente para as séries pluviométricas de Timbó, Pomerode, Luiz Alves e Blumenau. Para o município de Rio dos Cedros, com um total anual de 2.342,8 $\mathrm{mm}$, foi considerado um período Muito Chuvoso. Dos 66 extremos de precipitação verificados no ano de 2008, as cidades de Blumenau e Trombudo Central apresentam os maiores valores, com 9 e 8 extremos, respectivamente. A análise dos dados permitiu observar que as chuvas inferiores a $50 \mathrm{~mm}$ predominaram entre fevereiro e dezembro de 2008, o que justifica a ausência 
de um número relevante de extremos neste período. No mês de janeiro a estação pluviométrica encontrava-se inoperante. Em Rio do Sul não houve registro de extremos devido ao predomínio de precipitações inferiores a $50 \mathrm{~mm}$. Tais resultados mostram que em um mesmo ano podem haver períodos mais secos e mais chuvosos, sendo que a distribuição destas chuvas e a ocorrência de eventos extremos, mesmo isolados, são determinantes aos efeitos que podem causar. Dois anos diferentes podem ter totais anuais de chuva semelhantes, porém com distribuição distintas. No primeiro, a chuva acumulado ficou bem distribuída entre os meses, os dias; no segundo, o primeiro semestre pode ter sido seco e totais elevados de chuva serem registrados no segundo período.

O ano de 2011 apresentou totais anuais elevados. Foram 13 as estações que apresentaram anos muito chuvosos ou extremamente chuvosos. Os maiores valores acumulados foram encontrados nas estações pluviométricas de Pomerode $(2.753,4 \mathrm{~mm})$ e Rio dos Cedros $(2.661,5 \mathrm{~mm})$. Rio dos Cedros apresentou maior quantidade de dias com extremos de chuva (15), seguida de Luiz Alves e Pomerode com 12 registros cada. Os anos de 2002 e 2010 também apresentaram totais anuais elevados, sendo 2010 o ano de El Niño fraco. Em Taió, Pouso Redondo, Trombudo Central e Vidal Ramos o ano de 2002 foi classificado como Muito Chuvoso. Em Pomerode $(2.450,7 \mathrm{~mm}$ ), Apiúna $(1.975,7 \mathrm{~mm})$ e Blumenau $(2.070,2 \mathrm{~mm})$, o ano de 2010 foi caracterizado como um ano Muito Chuvoso e, em Rio dos Cedros, Extremamente Chuvoso, atingindo um total anual de 2.721,9 mm. No ano de 2002, não foram observados extremos de precipitação em Alfredo Wagner e em 2010 em Ituporanga, devido ao predomínio de precipitações com volumes diários inferiores a $50 \mathrm{~mm}$. Em 2002, o total de extremos foi de 67, enquanto no ano de 2010 foi de 106, sendo que os maiores extremos foram observados em Indaial, totalizando 11 registros de extremos de chuva. Em Timbó, o ano de 2001 foi considerado um ano Extremante Chuvoso com total anual de $2.077,7 \mathrm{~mm}$ e 6 registros de extremos no período. Em 2001, os maiores registros foram observados em Agorlândia (7).

As séries pluviométricas de Ituporanga e Alfredo Wagner apresentaram características diversas das demais estações. Em Ituporanga, além do ano de 1983 e 1990, o ano de 1961 se destaca como Extremamente Chuvoso. Em Alfredo Wagner, os anos de 1963 e 1973, foram classificados como extremamente chuvosos, sendo superados apenas pelo ano de 1983. A alta pluviosidade no ano de 1983 pode ser explicada pela ocorrência de um evento forte de El Niño, cujos efeitos foram observados em vários locais em todo o mundo. Em Brusque, os anos de 1961 e 1963 foram considerados extremamente chuvosos. O ano de 1963 foi um ano de El Niño fraco, o que poderia justificar o comportamento da precipitação no município. Neste ano o acumulado anual foi de $2.365,7 \mathrm{~mm}$ e acumulado mensal de $509 \mathrm{~mm}$ no mês de setembro, mês com os maiores valores de precipitação.

De modo geral, observar-se uma relação entre anos de alta pluviosidade e/ou com grande incidência de extremos e episódios de ENOS, como os anos de 1983, 1992, 1998, 2005 e 2010. Com exceção do ano de 2008, ano de La Niña, os anos de 1983 e 1998, correspondem a anos de forte El Niño. Anos com grande incidência de extremos, como anos de 1992 e de 2010, foram anos de El Niño, enquanto o ano de 2011, considerado de alta pluviosidade e com alta frequência absoluta de extremos, foi um ano de La Niña. 
A análise de frequência permite observar uma correspondência entre anos extremos de chuva e anos de alta pluviosidade, como os anos de 1983, 1990, 2010 e 2011. Anos de alta pluviosidade, como os anos de 1998 e 2008 não correspondem a anos de maiores valores de extremos. Neste caso, cabe ressaltar que inundações podem ocorrer em função da alta frequência de chuva (acumulando volume anual), como também de eventos extremos isolados, que podem contribuir para um volume anual alto.

Embora a classe $0,1-2,5 \mathrm{~mm}$ seja a mais comum, com ocorrência superior a 14,5\%, e as classes com valores diários >50 mm não chegam a ultrapassar $5 \%$ de frequência, evidenciando que extremos de precipitação são eventos de incidência mais rara, estes são os episódios que causam grandes prejuízos socioeconômicos, como os ocorridos em 1983 e 2008 na região do Vale do Itajaí, especialmente nas localidades de maior vulnerabilidade. Isso faz com que o conhecimento do comportamento da precipitação em uma região seja uma ferramenta importante para o planejamento em diversos setores da economia, uma vez que eventos naturais se convertem em danos sociais, construídos pela vulnerabilidade social.

\section{CONCLUSÕES}

Quanto à distribuição de chuvas, a bacia do rio Itajaí se caracteriza por apresentar valores normais $(\mathrm{Q}(0,5)$ ) entre $1.366,9 \mathrm{~mm}$ (Vidal Ramos) e $1.811,7$ $\mathrm{mm}$ (Pomerode). No centro-sul da bacia, valores normais variam entre $1.366,9$ $\mathrm{mm}$ (Vidal Ramos) e 1.560,4 mm (Alfredo Wagner), enquanto na porção lestenordeste, incluindo Luiz Alves, Pomerode, Rio dos Cedros, Timbó, Indail, Blumenau e Brusque, variam entre 1.611,1 mm (Timbó) e $1.811,7 \mathrm{~mm}$ (Pomerode). Valores normais, em torno de $1.600 \mathrm{~mm}$, foram encontrados em Agrolândia, a sudoeste da bacia. Quanto à intensidade, contata-se que a classe entre $0,1-2,5 \mathrm{~mm}$ é classe de maior ocorrência. Embora as classes entre 50-100 $\mathrm{mm}$ e $>100 \mathrm{~mm}$ não cheguem a $5 \%$ da frequência, contribuem para a ocorrência de eventos adversos em regiões suscetíveis a inundações e movimentos de massa, especialmente aquelas de maior vulnerabilidade social e econômica. Verifica-se a influência do fenômeno El Niño no regime de chuvas da bacia, devido à relação encontrada entre alta pluviosidade e/ou anos de grande incidência de extremos em anos de ENOS, como os anos de 1983, 1992, 1998, 2005 e 2010. No ano de 1983, na porção centro-leste da bacia, a precipitações anuais variaram entre $2862,9 \mathrm{~mm}$ (Rio dos Cedros) e $3.010,8 \mathrm{~mm}$ (Pomerode). Dessa forma, estes resultados podem contribuir na prevenção a desastres naturais, eventos recorrentes nas áreas urbanas da região em períodos chuvosos e/ou de extremos de chuva, especialmente as mais vulneráveis, assim como seus efeitos para a economia da região, especialmente na agricultura, como ocorrido em 1998, ano em que o excesso de chuvas afetou a agricultura catarinense, com quedas no rendimento e perda de qualidade da produção.

\section{REFERÊNCIAS}

ANEEL - Agência Nacional de Energia Elétrica. HIDRO-PLU Programa de Homogeneização de Dados Pluviométricos. Disponível em http://www2.ana.gov.br/Paginas/servicos/informacoeshidrologicas/monitoramen tohidro.aspx. Acesso em: 28 maio de 2017. 
BURGESS, Christopher P. et al. A macro-scale flood risk model for Jamaica with impact of climate variability. Natural Hazards, v.78, n.1, p.231-256, 2015.

CALVETTI, L.; BENETI, C.; GONÇALVES, J. E.; MOREIRA, I. A.; DUQUIA, C., BREDA, Â.; ALVES, T. A. Definição de classes de precipitação para utilização em previsões por categoria e hidrológica. In: XIV CONGRESSO BRASILEIRO DE METEOROLOGIA, 2006, Florianópois, Anais... Florianópolis, 2006.

CARVALHO, L. M. V.; JONES, C. Clima da Região Sul do Brasil. In: Iracema F. A. Cavalcanti et al. Org(s). Tempo e clima no Brasil. São Paulo: Oficina de Textos, p.259, 2009.

DIAS, M. A. F. S.; MINUZZI, R. B. As chuvas de novembro de 2008 em Santa Catarina: um estudo de caso visando a melhoria do monitoramento e da previsão de eventos extremos. Centro de Informações de Recursos Ambientais e Hidrometeorologia de Santa Catarina (CIRAM), 2009.

ELI K.; PITZ, J. W.; NEVES, L. de O.; HAVEROTH, R; E. C. de OLIVEIRA. Análise da distribuição da frequência de precipitação em diferentes intervalos de classes para rio do sul/sc. enciclopédia biosfera. Centro Científico Conhecer Goiânia, v.9, n.16, p.106, 2013.

ELI, K.; NEVES, L.; HAVEROTH, R.; PITZ, J. W.; PITZ I. W.; CATONI, J. M.; FRAGA, N. C. Clima, gestão do território e enchentes no Vale do Itajaí-SC. Terra Livre, v.1, n.20, p.159-170, 2015.

GONÇALVES, N. M. S. Impactos Pluviais e Desorganização do espaço Urbano em Salvador - BA. São Paulo: Tese (doutorado). Universidade de São Paulo, 2011.

MONTEIRO, J. B.; ROCHA, A. B.; ZANELLA, M. E. Técnica dos quantis para caracterização de anos secos e chuvosos (1980-2009): baixo curso do apodiMossoró/RN. Revista do Departamento de Geografia, v.23, p.232-249, 2012.

NERY, Jonas Teixeira. Dinâmica climática da região sul do Brasil. Revista Brasileira de Climatologia, v.1, n.1, 2005.

OLIVIERA, G. S.; SATYAMURTY, P.: O El Niño de 1997/98: Evolução e Impactos no Brasil. In: X CONGRESSO BRASILEIRO DE METEOROLOGIA, 1998, Brasília. Anais... Brasília, 1998.

NORIAH, A. B.; RAKHECHA, P. R. Probable maximum precipitation for $24 \mathrm{~h}$ duration over southeast Asian monsoon region-Selangor, Malaysia. Atmospheric research, v.58, n.1, p. 41-54, 2001.

RODRIGUES, M. L. G.; YNOUE, R.; ALVES, M. P. Episódios de chuva intensa na região da grande Florianópolis/SC: análise preliminar dos eventos e caracterização sinótica. In: IV SIMPÓSIO INTERNACIONAL DE CLIMATOLOGIA, 2011, João Pessoa. Anais... João Pessoa, Sociedade Brasileira De Meteorologia, 2011.

SALGADO, C. M.; DE OLIVEIRA PEIXOTO, M. N.; DE MOURA, J. R. da S. Caracterização espaço-temporal da chuva como subsídio à análise de episódios de enchentes no município de Angra dos Reis, RJ. Geosul, v.22, n.44, p. 7-26, 2007.

SATYAMURTY, P. MATTOS, L.F.; NOBRE, C.A.; SILVA DIAS P. L. Tropics - South America. In: Meteorology of the Southern Hemisphere, Ed. Kauly, D. J. and 
Vincent, D. G., Meteorological Monograph. American Meteorological Society, Boston, 119-139, 1998.

SORIANO, B. M. A.; GALDINO, S. Análise da distribuição da frequência mensal de precipitação para a sub-região da Nhecolândia, Pantanal, Mato Grosso do Sul, Brasil. Embrapa Pantanal. Boletim de Pesquisa e Desenvolvimento, 2002.

SOUZA, W. M.; DE AZEVEDO, P. V.; DE ARAÚJO, L. E. Classificação da Precipitação Diária e Impactos Decorrentes dos Desastres Associados às Chuvas na Cidade do Recife-PE. Revista Brasileira de Geografia Física, v.5, n.2, p.250268, 2012.

STRECK, N. A. Associação da variabilidade da precipitação pluvial em Santa Maria com a Oscilação Decadal do Pacífico. Pesquisa Agropecuária Brasileira, Brasília, v. 44, n.12, p.1553-1561, 2009.

TERACINES, Edson Baptista. Impactos econômicos do El Niño 97/98 na produção agrícola brasileira. In: IV SIMPÓSIO BRASILEIRO DE CLIMATOLOGIA GEOGRÁFICA, 2000, Rio de Janiro. Anais... Rio de Janeiro, v.4, 2000.

XAVIER, T. M. B. S.; SILVA, J. F. da; REBELLO, E. R. G. A técnica dos quantis e suas aplicações em Meteorologia, Climatologia e Hidrologia, com ênfase para as regiões brasileiras. Brasília: Thesaurus, 2002.

XAVIER, T. de M. B. S.; XAVIER, A. F. S. Binary Filtering for the Study of Persistence and Alternance of Dry and Wet Years in Northeast-Brazil. In: SYMPOSIUM ON METEOROLOGICAL ASPECTS OF TROPICAL DROUGHTS WITH EMPHASIS ON LONG-RANGE FORECASTING, Anais...p. 255-261, 1990.

XAVIER, T. de M. B. S.; XAVIER, A. F. S. Classificação de Anos Secos e Chuvosos na região Nordeste do Brasil e sua Distribuição Espacial, In: III CONGRESSO BRASILEIRO DE METEOROLOGIA, 1984, Belo Horizonte. Anais... Belo Horizonte, 1984.

XAVIER, T. de M. B. S.; XAVIER, A. F. S. Classificação e Monitoração de Períodos Secos e Chuvosos e Cálculo de Índices Pluviométricos para a região Nordeste do Brasil. Revista Brasileira de Engenharia/Cadernos de Recursos Hidricos, v.5, n. 2, p.7-31, 1987.

XAVIER, T. M. B. S.; XAVIER, A. F. S. Caracterização de períodos secos e ou excessivamente chuvosos no estado do Ceará através da técnica dos quantis: 1964-1998. Revista Brasileira de Meteorologia, v.14, n 2, p. 63-78, 1999.

ZANELLA, M. E. Impactos pluviais no Bairro cajuru - Curitiba - PR. Mercator, v. 10 , p. 93-105, 2007 